\title{
UNIVERSITYOF
}

FORWARD

THINKING

WESTMINSTER ${ }^{\text {用 }}$

WestminsterResearch

http://www.westminster.ac.uk/westminsterresearch

\section{Efficient Option Risk Measurement With Reduced Model Risk}

Mitra, S.

NOTICE: this is the authors' version of a work that was accepted for publication in Insurance: Mathematics and Economics. Changes resulting from the publishing process, such as peer review, editing, corrections, structural formatting, and other quality control mechanisms may not be reflected in this document. Changes may have been made to this work since it was submitted for publication. A definitive version was subsequently published in Insurance: Mathematics and Economics, 72, pp. 163-174, 2017.

The final definitive version in Insurance: Mathematics and Economics is available online at:

https://doi.org/10.1016/j.insmatheco.2016.09.006

(C) 2017. This manuscript version is made available under the CC-BY-NC-ND 4.0 license https://creativecommons.org/licenses/by-nc-nd/4.0/

The WestminsterResearch online digital archive at the University of Westminster aims to make the research output of the University available to a wider audience. Copyright and Moral Rights remain with the authors and/or copyright owners. 


\title{
Efficient Option Risk Measurement With Reduced Model Risk
}

\author{
Sovan Mitra*
}

*University of Liverpool,

Brownlow Hill,

Liverpool,

L69 3BX,

$U K$.

Tel: +44 1517942000

Email:sovan.mitra@liverpool.ac.uk 


\begin{abstract}
Options require risk measurement that is also computationally efficient as it is important to derivatives risk management. There are currently few methods that are specifically adapted for efficient option risk measurement. Moreover, current methods rely on series approximations and incur significant model risks, which inhibit their applicability for risk management.

In this paper we propose a new approach to computationally efficient option risk measurement, using the idea of a replicating portfolio and coherent risk measurement. We find our approach to option risk measurement provides fast computation by practically eliminating nonlinear computational operations. We reduce model risk by eliminating calibration and implementation risks by using mostly observable data, we remove internal model risk for complex option portfolios by not admitting arbitrage opportunities, we are also able to incorporate liquidity or model misspecification risks. Additionally, our method enables tractable and convex optimisation of portfolios containing multiple options. We conduct numerical experiments to test our new approach and they validate it over a range of option pricing parameters.
\end{abstract}

Key words: Options, model risk, option risk, replicating portfolio, risk measurement, delta method, efficient risk measurement, liquidity risk, arbitrage, portfolio optimisation, complex options, option trading strategies, static replication. 


\section{Introduction and Outline of Paper}

Computationally efficient risk measures of options are of paramount importance to research and industry, especially with the progressive increase in options trading and hedging. The events of the global credit crisis and past financial crises have demonstrated the necessity for adequate option risk management and measurement; poor risk measurement and management can result in bankruptcies and threaten collapses of an entire finance sector (see Kabir and Hassan (2005)). This is further exacerbated by the nonlinear losses associated with options and low margin requirements for options trading, which magnify losses.

Recently, there has been substantial literature on risk theory and risk measures, yet these have generally focussed on assets (e.g. stocks and bonds) rather than derivatives. Consequently, there is very little literature on option specific risk measurement. In order to measure the risk associated with an option we require the option's loss distribution. For the purpose of this paper let $\mathrm{Z}(\mathrm{t})$ denote the loss distribution associated with some asset or derivative. For example

$$
\mathrm{Z}(\mathrm{t})=\mathrm{C}(0)-\mathrm{C}(\mathrm{t}),
$$

where $\mathrm{C}(0)$ and $\mathrm{C}(\mathrm{t})$ represent the call option price at time now and time $\mathrm{t}$ respectively. We denote a risk measure by $\rho($.$) and measuring risk by \rho(Z)$.

As the option loss distribution is typically not available in a closed form solution, it must be obtained by Monte Carlo simulation. However, this can be computationally time consuming, even for the simplest option pricing models, because it requires computation of nonlinear functions (relating to the option pricing equation). Such long computation times are unsuitable for many financial applications e.g. high frequency trading. Consequently, this has led to the development of more computationally efficient methods of option risk measurement.

To improve the computation speed of option risk, the typical approach has been to apply some mathematical approximation to the option's loss distribution (e.g. Delta method). However, such computational improvements have been generally achieved at the cost of model risk, that is unforeseen losses associated with using a model e.g. calibration errors, implementation errors etc.. Since the purpose of such models are to measure or manage risk, such model risks defeat the purpose of the models and represents a significant issue.

Model risk is becoming increasingly important in risk management due to the increasing potential for it to cause significant losses; this has partly arisen due to the increasing reliance on models in the financial industry. For instance, model risk has been cited as a partial cause of the global financial crisis. Many institutions prefer to use models with lower model risk than models that are theoretically more consistent e.g. single factor interest rate models are preferred to multi-factor models due to their lower model risk. Although multi-factor models may be more realistic at explaining interest rate movements, they can result in higher estimation errors compared to single factor models.

In this paper we approach option risk measurement from a new direction. Rather than pursuing approximation methods (as has been done with prior methods for option risk measurement), we measure option risk using the risk of its equivalent replicating portfolio. This replicating portfolio method practically eliminates the requirement for calculating nonlinear operations for option risk and so provides faster computation times. Moreover, our replicating portfolio approach has lower model risk compared to competing computationally efficient option risk measurement methods. The replicating portfolio method does not admit arbitrage opportunities for portfolios containing put and 
call options (unlike other models), our method also has lower calibration risk, it can take into account liquidity risks and model misspecification, it can model the option risk of option portfolios without losing computational tractability and enables portfolio optimisation.

The outline of the paper is as follows: firstly we introduce option risk measurement and review current computationally efficient methods for measuring option risk. In the next section we then introduce our replicating portfolio approach to risk measurement. We then discuss the advantages of the replicating portfolio approach with respect to computational efficiency and model risk. We then conduct numerical experiments and finally end with a conclusion.

\section{Introduction to Option Risk Measurement and Literature Review}

In this section we introduce and review the literature on risk measurement, model risk, and computationally efficient option risk measurement.

\subsection{Risk Measurement and Model Risk}

A risk measure $\rho$ is a function mapping $Z$ to $\mathbf{R}$, that is

$$
\rho: Z \rightarrow \mathbf{R}
$$

We denote measuring risk by $\rho(Z)$. A popular industry risk measure is VaR (see Szego (2005)), that is $F(Z(t) \leq V a R)=\beta$, where $F($.$) is the cumulative probability distribution$ function and $\beta$ is a cumulative probability associated with threshold value $\mathrm{VaR}$, on the loss distribution of $\mathrm{Z}(\mathrm{t})$.

A significant milestone in risk measurement was achieved when Artzner et al. (1997) proposed the coherency axioms: axioms that risk measures $\rho($.) should obey to correctly measure risk. The coherency axioms are included in the Appendix for reference and further discussions on risk measures can be found in Goovaerts et al. (2004) and references therein.

To measure option risk we apply some risk measure to the loss distribution governing $C(0)-C(\delta t)$, where $C(\delta t)$ is the option value at some future time step $\delta t$. Whereas for stocks it is possible to analytically model the loss distribution in order to apply some risk measure, this is typically not possible for option loss distributions. Consequently, the option loss distribution of $C(0)-C(\delta t)$ must be obtained by computational methods (such as Monte Carlo simulation) and therefore the key difficulty in option risk measurement resides in obtaining the loss distribution in a computationally efficient approach. Once this distribution is obtained, we can apply a risk measure $\rho($.) to this distribution. For example, the VaR risk measure would determine the value associated with a cumulative probability $\beta$.

Currently, all option risk methods achieve computational efficiency in speed of computation by allowing model risk to increase. Model risk is defined as the risk of working with a potentially incorrect model, which leads to unexpected losses. Examples of model risks that can be incurred are increased calculation error, increased calibration errors or violation of fundamental theorems in Finance e.g. Law Of Arbitrage (to be addressed in later sections).

Model risk is a key problem in Finance; model errors can result in significant losses (e.g. Long Term Capital Management), they are playing an increasingly important role in industry and institutions are becoming ever more reliant on models for a variety of 
purposes. In option risk models, model risk is a particularly important issue because such models are used for risk management purposes. Hence it is important that such models have low model risks to prevent the models themselves incorrectly measuring risk or becoming a source of risk in themselves.

To give an example of model risk, the Delta-Gamma method (to be discussed later) should be theoretically always more preferable to the Delta method (to be discussed later) in calculating option risk. The Delta-Gamma method is a theoretically more accurate method than the Delta method, however the Delta-Gamma method requires calculation of European option parameter $\gamma$. As $\gamma$ may not be available in analytic form for many option pricing models, it can only be calculated by computational methods, which can distort calculation accuracy but also increase total computation time. In fact it should be noted that computationally evaluating second order partial differential equations in general (such as $\gamma$ ) can be inaccurate. Hence the model risk (and computational efficiency) of the Delta-Gamma method will be worse than the Delta method. Furthermore, the Delta-Gamma method removes the linear relation between the change in stock price $\delta S$ and change in call option price $\delta C$ (see later sections for more details), which significantly complicates valuing portfolios with options and portfolio optimisation (unlike in the Delta method).

The current literature on model risk is limited in finance. In Kerkhof et al. (2010), model risk is taken into account to determine capital reserves for banks. In particular, estimation risk, identification and misspecification models risks are addressed and combined with standard risk measures such as VaR. In Kondo and Saito (2012), a Bayesian method is proposed for measuring model risk for the insurance loss ratio. This method makes specific distribution assumptions and is focussed around VaR calculations, rather than application to any specific risk measure. In Alexander and Sarabia (2012) they develop a method for calculating model risk with respect to quantile risk measurement only. This allows institutions to adjust capital reserves to meet potential losses arising from model risk. In Schmeiser et al. (2012) analyse model risk with respect to solvency measures in the insurance sector.

Although there exists literature on model risk, the literature on model risk and computationally efficient option risk methods is non-existent to the best of our knowledge. The closest literature to address model risk with respect to option risk measurement is in Guillaume and Schoutens (2012), where model risk is investigated specifically with respect to calibration risk for vanilla and exotic options. However no reference is made with respect to computationally efficient option risk methods.

\subsection{Option Risk Measurement}

The current literature on option risk measurement is limited, particularly for computationally efficient methods. The most direct or "brute-force" approach to option risk measurement is the "full valuation method" (see Christoffersen (2003)). This involves Monte Carlo simulation of $S_{i}(\delta t)$ using some stock price model (e.g. geometric Brownian motion), where i denotes the index of the simulation sample. The option price value associated with $S_{i}(\delta t)$, that is $C_{i}(\delta t)$, is then calculated. The algorithm for the full valuation method is given in the Appendix for the Black-Scholes option pricing model $\mathrm{C}(\mathrm{S}(\mathrm{t}), \mathrm{t}, \mathrm{T}, \mathrm{r}, \sigma, \mathrm{K})$, which is also defined in the Appendix.

The advantages of the full valuation method is that firstly its accuracy can be improved as necessary; the accuracy of option risk calculation is always improved by increasing the number of simulations. Secondly, the full valuation method can be easily 
implemented, all that is required is Monte Carlo simulation of $S_{i}(\delta t)$ from some stock price model. The main disadvantage of the full valuation method is that it is a computationally expensive method (that is, it is a highly time consuming method); it requires computational calculations of $C_{i}(\delta t)$ for each simulated value $S_{i}(\delta t)$. The $C_{i}(\delta t)$ requires computing nonlinear terms (e.g. the Black-Scholes option pricing model requires calculating $\Psi\left(d_{1}\right)$ and $\Psi\left(d_{2}\right)$ ), which is computationally time consuming. Hence the computation of the option loss distribution is not computationally efficient, and inefficiency increases further for portfolios of options.

The high computation time incurred by the full valuation method has led to the development of alternative option risk methods with faster computation. One of the most popular option risk methods is the Delta method (see Britten-Jones and Schaefer (1998)). The call option's delta $\Delta(\mathrm{t})$ can give the option loss distribution of $\delta C$ by approximation:

$$
\begin{aligned}
& \Delta(t)=\frac{\partial C}{\partial S}, \\
& \Delta(t) \approx \frac{\delta C}{\delta S}, \\
& \therefore \delta \mathrm{C} \approx \Delta(\mathrm{t}) \delta \mathrm{S} .
\end{aligned}
$$

We obtain $\delta S$ by simulating $\mathrm{S}(\delta \mathrm{t})$ as we would under the full valuation method. The main advantage of the Delta method is that it has a significantly lower computation time than the full valuation method (see Christoffersen (2003)) as it mainly consists of computing linear operations, unlike the full valuation method. Additionally, the Delta method can generally be implemented quite easily, as $\Delta(\mathrm{t})$ is either available analytically, or can be easily evaluated by computational methods. A disadvantage of the Delta method is that the method is fundamentally dependent on an approximation of $\delta C$, therefore the accuracy of this method is fundamentally limited and cannot be improved by increasing Monte the number of simulations.

An alternative to the Delta method is the Delta-Gamma method, which uses a Taylor expansion to expand $\delta C$ up to squared terms (see Christoffersen (2003)). This gives:

$$
\delta C \approx \Delta \delta S+\frac{\gamma}{2}(\delta S)^{2}, \text { where } \gamma=\frac{\partial^{2} C}{\partial S^{2}} .
$$

The advantages of the Delta-Gamma method are that firstly it is theoretically more accurate than the Delta method. The Delta method only takes a Taylor expansion upto terms of power 1, whereas the Delta-Gamma method includes squared terms, and so will always be more accurate. Secondly, the Delta-Gamma method is computationally more efficient than the full valuation method. Although the Delta-Gamma method requires calculation of a nonlinear term $\gamma$, this is only required once for determining the entire option loss distribution, hence it is not a substantial increase in computation time. Furthermore, the option loss distribution requires calculation of the $(\delta S)^{2}$ term, for every sample; although this nonlinearity adds to the computational time it is still significantly less than the full valuation method.

The disadvantages of the Delta-Gamma method are firstly, it is fundamentally less accurate than the full valuation method. As the Delta-Gamma method is fundamentally 
dependent on an approximation of $\delta C$, the method's accuracy is fundamentally limited and cannot be improved by increasing Monte Carlo simulation. Secondly, the additional computation time required for the $(\delta S)^{2}$ terms means that it is not as computationally efficient as the Delta method. Finally, as mentioned before, the Delta-Gamma method requires $\gamma$ and this may not be analytically available, therefore computation of this second order partial differential can introduce model risks e.g. calculation errors.

In addition to the Delta and Delta-Gamma method, other less well-known option risk methods exist. In Sorwar and Dowd (2010) a simulation-lattice computational method is proposed. This enables one to estimate risk for various option positions, for a range of options (including exotic options and early exercise feature) as well as important underlying distribution features, such as heavy tails. However, such a computational method is computationally intensive and so does not offer fast computation, which is the focus of our paper.

In Hao and Yang (2011) option risk is measured but under the assumption of a regime switching stock price process. Also, the risk measurement is restricted to scenario based risk measures, hence its applications (and accuracy) are limited. In Broda (2012) computable expressions for risk are given, however this is restricted to the expected shortfall risk measure and that portfolios follow an elliptic multivariate t-distribution.

Other option risk measurements exist that apply approximation methods (hence also incur model risk). For example, one method is to apply the Cornish-Fisher approximation (see Christoffersen (2003)), where we assume the underlying return distribution is Gaussian with mean 0 and constant variance. Using a quadratic approximation we can obtain the first 3 moments of the distribution of $\delta C$, we can then approximately calculate VaR using a Cornish-Fisher approach. Other researchers have also applied moment matching and approximations to measure option risk by VaR e.g. see El-Jahel et al. (1999). The Delta-Gamma method has been developed in terms of a Cornish-Fisher expansion in Jaschke (2002); in Glasserman et al. (2001) Delta-Gamma is used to provide more efficient Monte Carlo simulated estimates of VaR; in Siven et al. (2009) Delta-Gamma is used along with Fourier inversions to calculate VaR.

\section{Option Risk Measurement by Replicating Portfolio}

As can be seen from the previous section, option risk methods are typically based on some approximation method and this can incur significant model risk. This is an important problem as such models are frequently used for risk management purposes. In this section, we show that we can measure option risk with a computationally efficient method by taking a different approach: using its replicating portfolio. This also provides significant model risk advantages.

In this section we first explain how our replicating portfolio method provides computational advantages in measuring option risk; we also show this method has computational advantages for a portfolio of options and portfolio optimisation. We also discuss key model risk advantages of our method, specifically put-call parity consistency, lower implementation risk, calibration risk and can take into account model misspecification and liquidity risk. It should also be noted that the replicating portfolio method can be applied to any contingent claim with a replicating portfolio and not just options. 


\subsection{Option Risk Calculation Method}

The key insight of Black and Scholes (1973) is that we can represent a European option by a replicating portfolio $\mathrm{V}(\mathrm{t})$, based on a no arbitrage argument. A replicating portfolio $\mathrm{V}(\mathrm{t})$ consists of $\phi_{1}(\mathrm{t})$ number of shares in the underlying of the option and $\phi_{2}(\mathrm{t})$ number of units in a riskless bond:

$$
V(t)=\phi_{1}(t) S(t)-\phi_{2}(t) B(t)
$$

where $\mathrm{B}(\mathrm{t})$ is the price of a riskless bond at time $\mathrm{t}$ (see Appendix for full equation). The negative sign for bonds means we short $\phi_{2}(\mathrm{t}) \mathrm{B}(\mathrm{t})$ bonds rather than purchase them. In the case of the Black-Scholes equation we have $\phi_{1}(t)=\Delta(t)$.

We achieve computationally efficient risk measurement of options by using its replicating portfolio for risk measurement and applying the coherency axioms. This allows the elimination of nonlinear operations in the computational calculation of option risk and so significantly reduces computation time. We now state this in our theorem.

Theorem 1 For a coherent risk measure $\rho($.$) the risk of an option, or any contingent$ claim, replicated by a replicating portfolio $\left(\Delta(t) S(t), \phi_{2}(t) B(t)\right) \quad$ is given by

$$
\rho(d C(t))=\Delta \rho(d S(t))+(\Delta S(t)-C(t)) r d t .
$$

Hence it can be seen from equation (3) that, excluding $\rho($.), the number of operations that are a nonlinear function of $d S(t)$ is zero.

\section{Proof:}

$$
\begin{aligned}
d C(t) & =\Delta(t) d S(t)-\varphi_{2}(t) d B(t) \text { by self-financing property, } \\
\rho(d C) & =\rho\left(\Delta(t) d S(t)-\varphi_{2}(t) d B(t)\right) \\
& =\rho(\Delta(t) d S(t))+\varphi_{2}(t) d B(t) \text { by translation invariance axiom, } \\
& =\Delta(t) \rho(d S(t))+\varphi_{2}(t) d B(t) \text { by homogeneity axiom, }
\end{aligned}
$$

since $d B(t)=r B(t) d t$, then we have

$$
\rho(d C(t))=\Delta(t) \rho(d S(t))+\phi_{2}(t) B(t) r d t
$$

substituting $\varphi_{2}(t) B(t)=\Delta(t) S(t)-C(t)$, we have

$$
\rho(d C(t))=\Delta(t) \rho(d S)+(\Delta(t) S(t)-C(t)) r d t
$$

To be able to understand our method it is important to understand the variables that are functions of $\mathrm{S}(\delta \mathrm{t})$, since such (non-linear) functions significantly increase computation time as they must re-calculated for every simulated value of $S(\delta t)$. This is 
achieved by understanding the principles relating to a replicating portfolio, namely no arbitrage and self-financing.

The replicating portfolio $\mathrm{V}(\mathrm{t})$ is an adapted process to $\mathrm{C}(\mathrm{t})$; it has identical values to $\mathrm{C}(\mathrm{t})$ for all $\mathrm{t}$, assuming the market is arbitrage free (see the Appendix for a definition). Therefore

$$
\mathrm{C}(\mathrm{t})=\mathrm{V}(\mathrm{t}), \forall \mathrm{t} \leq \mathrm{T}
$$

This also implies the risk of $\mathrm{V}(\mathrm{t})$ and $\mathrm{C}(\mathrm{t})$ must be identical because their loss functions must be identical. In other words, we have:

$$
\rho(C(t))=\rho(V(t)), \forall t \leq T \text {. }
$$

A replicating portfolio also has the important property that it must be self-financing. This is normally not a crucial issue in option theory, however for our option risk measurement method it is crucial to the derivation. By self-financing we have:

$$
\begin{aligned}
\mathrm{dV}(\mathrm{t}) & =\phi_{1}(\mathrm{t}) \mathrm{dS}(\mathrm{t})-\phi_{2}(\mathrm{t}) \mathrm{dB}(\mathrm{t}), \\
& =\phi_{1}(\mathrm{t}) \mathrm{dS}(\mathrm{t})-\phi_{2}(\mathrm{t}) \mathrm{rB}(\mathrm{t}) \mathrm{dt} .
\end{aligned}
$$

In terms of option risk, the key part of equation (8) is that neither $\phi_{1}(\mathrm{t})$ nor $\phi_{2}(\mathrm{t})$ change when we calculate $\mathrm{dV}$ (or $\mathrm{dC}$ ), for they are constant. Therefore to determine the option loss distribution associated with $\mathrm{dC}$ we do not need to calculate $\phi_{1}(\mathrm{t})$ and $\phi_{2}(\mathrm{t})$ for each simulated value of $S(\delta t)$. This is because both $\phi_{1}(t)$ and $\phi_{2}(t)$ are functions of $S(0)$ but not $\mathrm{S}(\delta \mathrm{t})$. If $\mathrm{V}(\mathrm{t})$ were not a self-financing portfolio then we would have under standard differentiation (see Kwok (1998))

$$
d V(t)=\phi_{1}(t) d S(t)-\phi_{2}(t) d B(t)+d \phi_{1}(t) S(t)-d \phi_{2}(t) B(t)
$$

This equation would significantly complicate computational calculation of option price changes because we would need to simulate changes in $d \phi_{1}$ and $d \phi_{2}$, in addition to dS. In such a case it may be better to use the full valuation method instead.

The reasons that both $\phi_{1}(\mathrm{t})$ and $\phi_{2}(\mathrm{t})$ are functions of $\mathrm{S}(0)$ but not $\mathrm{S}(\delta \mathrm{t})$ in equation (8) are financial and mathematical. Mathematically the theory is related to forward differences in stochastic differentials (the reader is referred to Bjork (2004) for a thorough discussion). Essentially, if we were to discretise equation (9) we would have (Jarrow and Turnbull (1996))

$$
\delta \mathrm{V} \approx \phi_{1}(\mathrm{t})(\mathrm{S}(\mathrm{t}+\delta \mathrm{t})-\mathrm{S}(\mathrm{t}))-\phi_{2}(\mathrm{t}) \mathrm{rB}(\mathrm{t}) \delta \mathrm{t} .
$$

At time $\mathrm{t}$ we have only observed $\mathrm{S}(\mathrm{t})$ and not $\mathrm{S}(\mathrm{t}+\delta \mathrm{t}) ; \phi_{1}(\mathrm{t})$ remains unchanged during the time period $\mathrm{t}$ to $\mathrm{t}+\delta \mathrm{t}$. After time $\mathrm{t}+\delta \mathrm{t}$ (so $\delta \mathrm{t}$ has elapsed), $\mathrm{S}(\mathrm{t}+\delta \mathrm{t})$ has been observed and then we adjust the number of shares and bonds to give the new values $\phi_{1}(\mathrm{~S}(\mathrm{t}+\delta \mathrm{t}), \mathrm{t}+\delta \mathrm{t}) \quad$ and $\phi_{2}(\mathrm{~S}(\mathrm{t}+\delta \mathrm{t}), \mathrm{t}+\delta \mathrm{t}) \quad$. From a financial point of view, we cannot have $\phi_{1}(\mathrm{t})$ (or $\phi_{2}(\mathrm{t})$ ) changing until we observe $\mathrm{S}(\mathrm{t})$ because the number of stocks and bonds we trade depend on the stock price we actually observe now. 
In conclusion we can say that $\Delta(\mathrm{t}), \mathrm{C}(\mathrm{t})$ and $\mathrm{S}(\mathrm{t})$ are not functions of $\mathrm{dS}$ (and so they are constant in $\mathrm{dC}$ ) due to the self-financing property. Hence in calculating $\rho(\mathrm{dC}(\mathrm{t}))$ we do not need to re-calculate them or perform nonlinear operations for each simulated value of dS. In fact other than calculating the option $\Delta(\mathrm{t})$ there are no nonlinear operations and $\Delta(t)$ is only calculated once during the entire simulation, hence does not represent a significant increase computation time. The replicating portfolio method is therefore a computational efficient method of calculating option risk.

It is also worth pointing out that $\Delta(\mathrm{t})$ is generally calculated for contingent claims even if no risk measurement is conducted, hence it generally imposes no additional computational time or analytical 'cost'. We also note that $\Delta(\mathrm{t})$ and $\phi_{2}(\mathrm{t})$ represent the number of units of stocks and bonds respectively (and are constants). hence we can apply the homogeneity axiom and take them outside $\rho($.$) (see equation (7)). They are also$ normally given in analytic form and so do not increase computation time (alternative expressions for them are given in the Appendix).

The replicating portfolio method is also able to achieve computational efficiency without sacrificing accuracy. The replicating portfolio option risk measure is based on equation (9); this equation is an identity for $\mathrm{dC}$, therefore it is identical to $\mathrm{dC}$ for all states of the world and is not an approximation. We can therefore always increase accuracy by increasing the number of simulations and reducing $\delta t$ to produce results of $\delta C$ equivalent to that of the full valuation method. On the other hand, to increase the accuracy of the full valuation method involves increasing the number of simulations and so the number of nonlinear operations, which is computationally expensive.

The Delta method (and other methods) are fundamentally limited in accuracy because they are approximations. For instance, the Delta and Delta-Gamma methods are taken from an approximation of the Taylor series expansion of $\delta \mathrm{C}$; in order to achieve full accuracy we require the Taylor series to an infinite series expansion with increasingly more nonlinear terms (which increases computation time). The Delta and Delta-Gamma methods will therefore never reach as accurate a calculation of $\delta \mathrm{C}$ as that of the full valuation method, regardless of the number of simulations executed. Such inaccuracies can be particularly important in high volume trading (e.g. high frequency trading), where minor inaccuracies can lead to magnified and cause unforeseen trading losses.

\subsection{Portfolios with Options: Option Risk Measurement and Portfolio Optimisation}

We would like to be able to value the change in value of a portfolio containing options, so that we could obtain the portfolio loss distribution and so measure its risk. Additionally portfolios frequently require rebalancing, that is optimising the weighting of assets and derivatives in the portfolio, to optimise some metrics (e.g. risk, expected etc.). Consequently, portfolio models are not only required for risk measurement but also for enabling optimisation.

In Christoffersen (2003) the Delta and Delta-Gamma methods are examined in terms of their use for portfolio risk measurement, when a portfolio contains stocks and options on the same stock (i.e. the underlying). In Christoffersen (2003) a portfolio D(t) containing $\mathrm{n}$ units of a stock and an option on the same stock (underlying) is defined by

$$
\mathrm{D}(\mathrm{t})=\mathrm{nS}(\mathrm{t})+\mathrm{C}(\mathrm{S}(\mathrm{t}))
$$


We now model the same portfolio $\mathrm{D}(\mathrm{t})$ using the replicating portfolio method (instead of the Delta and Delta-Gamma methods) and show that the replicating portfolio method is computational efficient as well as has benefits for portfolio optimisation (unlike the DeltaDelta-Gamma methods).

Using the replicating portfolio method to model the change in the portfolio's value $\delta \mathrm{D}(\mathrm{t})$ we have

$$
\delta \mathrm{D}(\mathrm{t}) \approx(\mathrm{n}+\Delta) \delta \mathrm{S}(\mathrm{t})+\phi_{2} \delta \mathrm{B} .
$$

For a full detail of the proof, please see the Appendix. Hence the change in the portfolio's value involves linear operations and so is not computationally expensive. If we were to use the Delta-Gamma method then we would have $(\delta S)^{2}$ terms in the $\delta \mathrm{D}(\mathrm{t})$ expression and so would incur higher computation time (due to the calculation of nonlinear terms). Alternatively, using the Delta method would be computationally more efficient than the Delta-Gamma method, however it would be less accurate than the replicating portfolio method.

If $\mathrm{D}(\mathrm{t})$ is extended to include a set of $\mathrm{n}$ options with different $\mathrm{K}$ and $\mathrm{T}$ (but on the same underlying stock), that is

$$
\mathrm{D}(\mathrm{t})=\sum_{\mathrm{i}=1}^{\mathrm{n}} \mathrm{C}_{\mathrm{i}}\left(\mathrm{S}(\mathrm{t}), \mathrm{K}_{\mathrm{i}}, \mathrm{T}_{\mathrm{i}}\right)
$$

then by the replicating portfolio method we have

$$
\delta \mathrm{D} \approx \sum_{\mathrm{i}=1}^{\mathrm{n}} \phi_{1 \mathrm{n}} \delta \mathrm{S}+\phi_{2 \mathrm{n}} \delta \mathrm{B} .
$$

Now if we assume we have a more complex portfolio:

$$
D(t)=\sum_{i=1}^{n} v_{i} S_{i}+\sum_{j=1}^{m} v_{j} C_{i}\left(S_{i}(t), K_{i}, T_{i}\right),
$$

where $v_{i}$ and $v_{j}$ represent the number of units stocks and options, respectively, and $\mathrm{m}$ equals the total number of different stocks. Additionally, if we apply the same modelling assumptions used in the Delta method for modelling such portfolios then we assume all stocks and options are uncorrelated. Therefore the replicating portfolio approach gives

$$
\delta \mathrm{D}(\mathrm{t}) \approx \sum_{\mathrm{i}=1}^{\mathrm{n}} \mathrm{v}_{\mathrm{i}} \delta \mathrm{S}_{\mathrm{i}}+\sum_{j=1}^{\mathrm{m}} \mathrm{v}_{\mathrm{j}} \Delta_{\mathrm{j}} \delta \mathrm{S}_{\mathrm{j}}(\mathrm{t})+\phi_{2 j} \delta \mathrm{B} .
$$

The replicating portfolio method is computationally more efficient than if we used the Delta-Gamma method (since this would involve non-linear terms). The replicating portfolio method is more computational efficient as we do not need to re-calculate any non-linear terms with each simulation. Similarly, the Delta method may be marginally more efficient but it would lead to more inaccurate valuation of $\delta \mathrm{D}(\mathrm{t})$. 
In addition to efficient risk measurement of portfolios with options, the replicating portfolio method offers computational advantages in portfolio optimisation. Specifically, for a portfolio $\mathrm{L}(\mathrm{t})$ containing $\mathrm{N}$ stocks and $\mathrm{M}$ options, we would like to optimise

$$
\max _{w_{i}, w_{j} \forall i, j} f(d L(t))=\sum_{i=1}^{N} w_{i} d S_{i}(t)+\sum_{j=1}^{M} w_{j} d C_{j}(t),
$$

where $w_{i}, w_{j}$ are the stock and option weights respectively. The inclusion of options in $\mathrm{L}(\mathrm{t})$ means the optimisation of $\mathrm{f}(\mathrm{dL}(\mathrm{t}))$ is nonconvex, and therefore a non-trivial optimisation. Firstly there exist fewer algorithms for nonconvex optimisation, so there may not exist an optimisation method. Secondly, nonconvex optimisation implies that an optimal solution may only be a locally optimal solution, rather than a globally optimal solution.

If one were able to replace options with a linear expression then one would have a linear optimisation, which is highly desirable as they enable powerful and well-developed algorithms to be applied (such as linear programming and stochastic programming) to large portfolios. Linear optimisation of $\mathrm{L}(\mathrm{t})$ is possible by using the replicating portfolio approach to options. Therefore we would have:

$$
\begin{gathered}
\max _{w_{i}, w_{j} \forall i, j} f(d L(t))=\sum_{i=1}^{N} w_{i} d S_{i}(t)+\sum_{j=1}^{M} w_{j}\left(\Delta_{j}(t)\left(d S_{j}(t)\right)+\left(\Delta_{j}(t) S_{j}(t)-C_{j}(t)\right) r d t\right), \\
=\sum_{i=1}^{N} w_{i} d S_{i}(t)+\sum_{j=1}^{M} w_{j} \Delta_{j}(t) d S_{j}(t) .
\end{gathered}
$$

The last line is possible because $\left(\left(\Delta_{j}(t) S_{j}(t)-C_{j}(t)\right) r d t\right)$ is a constant and so does not affect the optimisation (other than in the possible case there are linear constraints imposed in the optimisation).

\subsection{Calibration and Implementation Risk}

A key model risk that is frequently incurred in models is calibration risk, that is unexpected losses arising from incorrect model calibration. For instance, the model is calibrated using bias data, or the calibration method itself leads to inaccurate modelling (for example minimising least squares error in linear regression is known to lead to inaccurate modelling if outliers exist in the calibration data). In fact in industry, local volatility models are preferred to stochastic volatility models due to their lower calibration risk.

Another important area of model risk is implementation risk, that is unexpected losses arising from implementing the model. In order to utilise a model it requires some method of implementation, typically using a computational program, and this introduces some risk as the implementation may introduce some unexpected errors in calculation. For example, in implementing a model in a program one may be required to calculate the Normal cumulative probabilities and this requires some method to calculate the probabilities (e.g. using a table, applying some approximation of the cumulative distribution function, etc.). Consequently, some models are not used in industry due to the implementation risks involved (e.g. using models with unusual probability distributions may be difficult to implement in computer programs). 
We will now explain how our replicating portfolio method has significant model risk advantages compared to other computationally efficient option risk methods.

\subsubsection{General Calibration and Implementation Risk}

For the replicating portfolio method, other than $\Delta(\mathrm{t})$ the remaining parameters in equation (3) (that is $\mathrm{S}(\mathrm{t}), \mathrm{C}(\mathrm{t})$ and $\mathrm{r}$ ) are observable variables and so the method does not require calibration to any data or any calculation (we define an observable variable to mean a variable whose value can be observed in the market or 'real world' without requiring calibration of models to calculate its value e.g. $\mathrm{S}(\mathrm{t}), \mathrm{T}$ and $\mathrm{r}$ ). Furthermore the calculation of $\Delta(\mathrm{t})$ is a function of observable variables (except volatility). Therefore the number of parameters that could cause model risk are significantly limited; our method requires no more observability than the observability required for the Black-Scholes model itself, which is considered a highly observable model. Additionally, the limited calibration required increases the stability of calibration of the model, that is the model will not require frequent re-calibration to enable realistic model forecasting.

Our replicating portfolio method reduces implementation risk by its tractable computational implementation. The method requires usage of observable variables and just 1 calculation for $\Delta(\mathrm{t})$, and this is a computationally tractable calculation. It can be numerically evaluated easily (e.g. binomial trees or finite difference methods), even for non-trivial $\mathrm{S}(\mathrm{t})$ processes, and with sufficient level of accuracy. For example, for an American option we can easily calculate the $\Delta(\mathrm{t})$ using a binomial tree method. The $\Delta(\mathrm{t})$ calculation would not form part of the Monte Carlo simulation; the $\Delta(\mathrm{t})$ is always a oneoff calculation and so the replicating portfolio method still remains computationally efficient. For a basket option the replicating portfolio method only requires calculation of $\phi_{1}$ for each asset in the portfolio (and $\phi_{2}$ can be deduced using equations (26) and (25)).

To implement the replicating portfolio method we require $\Delta(\mathrm{t})$ and this is generally analytically possible to determine for a range of option pricing models. Other risk measuring methods are not as easy to implement. For instance, the Delta-Gamma method requires calculating $\gamma$ and this is a second order partial derivative. Such a derivative may not be easily available and may be analytically intractable to derive, especially for complex contingent claims or non-trivial $\mathrm{S}(\mathrm{t})$ processes. Furthermore, it is well known that numerical computation can be intractable for second order partial derivatives, leading to inaccurate calculations. In the case of a basket option the Gamma method requires a second order partial differential equation for each asset in the portfolio (by applying multivariate Taylor's Theorem), which can become intractable for large portfolios.

The replicating portfolio method reduces implementation risk further as it is also analytically more tractable compared to other methods, in particular we can analytically derive $\rho(d C)$ from $\rho(d S)$ and using equation (3). For instance, we can easily calculate VaR using equation (3):

$$
\operatorname{VaR}(\mathrm{dC}(\mathrm{t}))=\Delta(\mathrm{t}) \operatorname{VaR}(\mathrm{dS}(\mathrm{t}))+(\Delta(\mathrm{t}) \mathrm{S}(\mathrm{t})-\mathrm{C}(\mathrm{t})) \operatorname{rdt}
$$

(we note that VaR only fails as a coherent risk measure in terms of subadditivity, hence we can apply the translation invariance axiom to $\mathrm{VaR}$ to obtain this equation). Furthermore, if we assume dS follows geometric Brownian motion then VaR will be the VaR for a Gaussian distribution (for which many analytic equations exist), multiplied by $\Delta(t)$, with its centre shifted by the drift term and the expression $(\Delta(t) S(t)-C(t)) r d t$. 
If one were to apply another method to measure option risk, we would not necessarily be able to derive analytical solutions for any given risk measure. For eample, if we wished to determine VaR using the Delta-Gamma method then

$$
\operatorname{VaR}(\mathrm{dC})=\operatorname{VaR}\left(\Delta \delta \mathrm{S}+\frac{\gamma}{2}(\delta \mathrm{S})^{2}\right)
$$

This would not be a tractable method of measuring VaR (and similarly for other risk measures). Firstly, the measurement of $\operatorname{VaR}(\mathrm{dC})$ is a function of $\gamma$, which can difficult to accurately determine for options. Secondly, the VaR measurement is now on a non-trivial distribution: the distribution obtained from adding the distributions of $\delta S$ and $(\delta S)^{2}$. There may not exist any analytical solution for the overall distribution, let alone the VaR equation (or any risk measure). Furthermore, computational implementation to obtain VaR or any other risk measure would be computationally expensive.

Finally, the replicating portfolio method reduces implementation risk and general model risk by having a parsimonious model with few modelling assumptions. The replicating portfolio method is based on an identity for $\delta C$ using the self-financing property and arbitrage free assumption (both of these are not restrictive assumptions). Our method is not restricted to any risk measure, particular to any assets or distributions. Other risk measurement methods make restrictive assumptions about stock price distributions, variables (e.g. state of the economy) and apply to particular risk measures only (e.g. VaR).

\subsubsection{Extreme Values: Calibration and Implementation Risk}

Using the replicating portfolio method, the estimation error will be lower for extreme values compared to other methods. It is important to be able to measure the risk of extreme losses, however, measuring and managing risk under extreme values poses a number of significant problems. Firstly, many option risk measures cannot value at extreme values because they are only valid over small changes e.g. the Delta method. Secondly, there may not exist sufficient observations to confidently estimate extreme values; this is particularly the case for extreme values as such extreme events tend to occur rarely. Finally, the inability to accurately fit or estimate distributions for extreme values means that we cannot provide an realistic models for extreme values (see Dowd (2011)).

In risk management theory, one applies EVT (extreme value theory) to determine the risk of extreme values on stocks. Therefore, our replicating portfolio method can also obtain risk measures on extreme values of options, by applying EVT to stock prices. In other words, from equation (3) it can be seen that we can obtain extreme risk measurement values of $\rho(\mathrm{dC})$ from extreme risk measurement of $\rho(\mathrm{dS})$.

If we were using other models we would not necessarily be able to obtain extreme values for option risk in the same way. For instance in the Delta-Gamma model, to obtain extreme value measurements in $\mathrm{dC}$ we would require simulations of $\mathrm{dS}$ and $\mathrm{dS} \mathrm{S}^{2}$, hence any estimation errors in simulation would be squared. Such errors would be magnified further when calculating extreme values using Extreme Value Theory. Additionally, we must multiply the $d S^{2}$ term by $\gamma$, which is a partial derivative and so is difficult to accurately compute or estimate, leading to higher potential extreme value errors. 


\subsection{Arbitrage Free Option Modelling: Put-Call Parity Consistency}

The put-call parity is an important theorem between calls $\mathrm{C}(\mathrm{S}(\mathrm{t}), \mathrm{t}, \mathrm{T}, \mathrm{r}, \mathrm{K})$ and puts $\mathrm{P}(\mathrm{S}(\mathrm{t}), \mathrm{t}, \mathrm{T}, \mathrm{r}, \mathrm{K})$; an explanation is given in the Appendix. The put-call parity holds under a range of conditions, it is a model independent requirement, and its violation implies arbitrage between puts and calls (a serious mispricing and hence it is generally not violated). The put-call parity has important industry applications because it is frequently used in industry to value puts because quoted put prices are normally not available; using quoted call prices and the put-call parity we can value put prices.

The put-call parity is also important to enable correct valuation of portfolios containing a range of options e.g. in option trading strategies and static replication methods (see for instance Ma et al. (2016)). Option trading strategies (e.g. a butterfly, a strip and a strangle to name a few) involve purchasing a range of put and call options on the same underlying (see Hull (2000)). This portfolio of options is bought in such a way as to construct a net position that will benefit from a particular movement in the underlying. Static replication involves using a portfolio of plain vanilla European puts and calls to hedge an exotic derivative (see Derman et al., (1995)). Both option trading strategies and exotic derivatives hedging are becoming increasingly popular in industry and so also important to risk manage.

There exist option risk methods that violate the put-call parity, specifically the Delta and the Delta-Gamma methods (two of the most popular option risk models), and so allow arbitrage opportunities. We will now prove this.

Lemma 1 For any given underlying and any option pricing model, the Delta and DeltaGamma methods do not obey the put-call parity. Therefore the Delta and Delta-Gamma methods admit arbitrage opportunities in porfolios containing at least any two of the following: put option, call option or shares in the underlying.

Proof:

By Delta-Gamma method we have

$$
\delta \mathrm{P} \approx \Delta_{\mathrm{p}} \delta \mathrm{S}+\frac{\gamma^{2}}{2}(\delta \mathrm{S})^{2}
$$

where $\Delta_{p}$ is the option delta for a put.

By the put-call parity we also have

$$
\begin{gathered}
\mathrm{P}=\mathrm{Ke}^{-\mathrm{r}(\mathrm{T}-\mathrm{t})}-\mathrm{S}(\mathrm{t})+\mathrm{C}, \\
\mathrm{dP}=\mathrm{dC}+\mathrm{d}\left(\mathrm{Ke}^{-\mathrm{r}(\mathrm{T}-\mathrm{t})}\right)-\mathrm{dS}, \\
\delta \mathrm{P} \approx \delta \mathrm{C}+\delta\left(\mathrm{Ke}^{-\mathrm{r}(\mathrm{T}-\mathrm{t})}\right)-\delta \mathrm{S} .
\end{gathered}
$$

By the Delta-Gamma method we can express $\delta C$ as

$$
\delta \mathrm{C} \approx \Delta \delta \mathrm{S}+\frac{\gamma^{2}}{2}(\delta \mathrm{S})^{2}
$$

Also for any option pricing model it is known that

$$
\Delta_{p}=\Delta-1
$$


Now if we substitute $\delta C$ from equation (14) into equation (13) then we have

$$
\begin{aligned}
\delta \mathrm{P} & \approx\left(\Delta \delta \mathrm{S}+\frac{\gamma^{2}}{2}(\delta \mathrm{S})^{2}\right)+\delta\left(\mathrm{Ke}^{-\mathrm{r}(\mathrm{T}-\mathrm{t})}\right)-\delta \mathrm{S}, \\
& \approx \delta \mathrm{S}(\Delta-1)+\frac{\gamma^{2}}{2}(\delta \mathrm{S})^{2}+\delta\left(\mathrm{Ke}^{-\mathrm{r}(\mathrm{T}-\mathrm{t})}\right), \\
& \approx \delta \mathrm{S} \Delta_{\mathrm{p}}+\left(\gamma^{2} / 2\right)(\delta \mathrm{S})^{2}+\delta\left(\mathrm{Ke}^{-\mathrm{r}(\mathrm{T}-\mathrm{t})}\right) \text { by equation }(15)
\end{aligned}
$$

Hence equation (10) and equation (18) are not equal. Therefore put-call parity does not exist and so there exists arbitrage.

Remark 1 The Delta method is a special case of the Delta-Gamma method and so by the same proof it can be seen that the Delta method does not have put-call parity. Therefore the Delta method also allows arbitrage opportunities.

An explanation of the proof is as follows: if the Delta-Gamma method obeyed the putcall parity then substitution of an equation or expression from the put-call parity equation should give the same equation for $\delta P$, that is equations (10) and equation (18) should be equal. However these 2 equations are not equal and so this implies the put-call parity is not obeyed. An example of the Delta-Gamma method giving arbitrage opportunities in the put-call parity is given in the Appendix.

The replicating portfolio method must obey the put-call parity by construction and so does not admit arbitrage opportunities arising from this (for completeness we give the proof in the Appendix). The inability for some option risk methods to obey the put-call parity has significant consequences upon the applicability and risk management. Firstly, it can allow arbitrage opportunities to occur; incorrect models will not be able to detect incorrect prices according to the put-call parity, allowing serious mispricing to occur.

Secondly, incorrect models encourage 'internal' arbitrage opportunities (see Alexander (2001)). This is when 1 department within an institution takes advantage of the mispricing of derivatives and securities by another department (within the same institution). This enables one department to make riskless profits at the expense of another department making a riskless loss; such weakening of a department by another department (within the same institution) does not lead to overall profits for the institution and so is not a productive activity. The replicating portfolio method eliminates the possibility of internal arbitrage opportunities as it will always guarantee obeying the putcall parity, unlike the Delta or Delta-Gamma methods.

Finally, option risk methods that do not obey the put-call parity can give different risk measurements on the same portfolio. For instance, in equations (28) and (29) we have 2 different values for exactly the same option, which would give 2 different risk measurements for the same option. This can lead to inconsistent risk management of the same portfolio.

\subsection{Model Misspecification}

Model misspecification is becoming an increasing important factor in model risk. An example of model misspecification would be to model volatility as a constant (instead of varying with time) and so lead to incorrect values of volatility at different points in time. A popular method for addressing misspecification is the banded parameter model (see Wilmott et al. (1998)); in this model we subsume the misspecification into an appropriate variable and allow this variable's value to vary between a maximum and minimum limit. For example, if we choose the variable volatility then its value will be allowed to vary between the limits $\sigma^{-}<\sigma<\sigma^{+}$; alternatively we could have chosen $\mathrm{r}$ so that $\mathrm{r}$ would be bounded $r^{-}<r<r^{+}$. The banded model is also useful because we can determine worst and 
best case scenarios for option risk, which are important as they are frequently used in risk management.

We would like to be able to use the banded parameter model in option risk modelling to take into account model misspecification risk. To achieve this, we must be aware that the banded parameter model is only applicable if the model assumes the no arbitrage conditions. Therefore we can apply the replicating portfolio method to option risk to the banded parameter model because it is based on no arbitrage conditions.

For the purposes of option risk measurement we will restrict our attention to varying volatility in bands (first proposed by Avellaneda et al. (1995)), rather than any other variable, because it is a common source of model misspecification. In the banded parameter model, the worst and best case scenarios are not simply obtained by using the lowest and highest volatility values but by applying the arbitrage principles.

The Black-Scholes equation is derived on the assumption that it constructs a riskless hedge; for a call option we have

$$
\mathrm{dC}-\Delta \mathrm{dS}=-\phi_{2} \mathrm{~dB}
$$

Now to avoid arbitrage opportunities we assume the return on the worst case replicating portfolio earns the riskless rate, that is

$$
\begin{array}{r}
\min _{\sigma^{-}<\sigma_{<\sigma^{+}}}(\mathrm{dC}-\Delta \mathrm{dS})=-\phi_{2} \mathrm{~dB}, \\
\min _{\sigma^{-}<\sigma<\sigma^{+}} \mathrm{d} \Pi=-\phi_{2} \mathrm{~dB},
\end{array}
$$

where $\mathrm{d} \Pi=\mathrm{dC}-\Delta \mathrm{dS}$. Our objective is

$$
\min _{\sigma^{-}<\sigma<\sigma^{+}}\left(\frac{\partial C}{\partial t}+\frac{\sigma^{2} S^{2} \gamma}{2}\right)
$$

It can be shown that we minimise $\mathrm{d} \Pi$ if $\sigma=\sigma^{+}$for $\gamma>0$ and $\sigma=\sigma^{-}$for $\gamma<0$. Therefore to find the best case option risk measurement we use $\sigma=\sigma^{+}$if $\gamma<0$ and $\sigma=\sigma^{-}$if $\gamma>0$; for the worst case option risk measurement we would use $\sigma=\sigma^{+}$for $\gamma>0$ and $\sigma=\sigma^{-}$for $\gamma<0$.

As discussed before, some models do not obey the no arbitrage assumption (e.g. under put-call parity). Consequently, models such as Delta and Delta-Gamma cannot be applied to the banded parameter method for model misspecification. Therefore the Delta and Delta-Gamma methods do not allow us to determine best and worst case scenarios for option risk measurement, or take into account model misspecification risks.

\subsection{Liquidity Risk}

An increasingly important component of model risk is liquidity risk (see for example Acharya et al. (2015)), which is essentially the risk of transaction costs (T) increasing. Transaction costs can form a significant part of risk because they can substantially increase the losses incurred in trading, they can also vary with trading volume, the state of the economy and the market size to name a few factors.

Although there exist many liquidity models for stocks, currently there do not exist many liquidity models for options. One model by Krakovsky (1999) prices liquidity costs into options by modifying the partial differential equation governing the option pricing equation. However the resulting partial differential equation has no analytic solution, so it must be solved computationally, which is computationally expensive. Krakovsky 
(1999) model also ignores bid-ask spreads changing with time, which is an important factor in liquidity risk.

One popular and well known liquidity model is Leland (1985) transaction cost model. Using the replicating portfolio method of option risk modelling it is possible to take into account liquidity risk using Leland's model. This is possible because Leland's model is based on the replicating portfolio principle, unlike other option risk models. In Leland's model the transaction costs $\mathcal{T}$ are proportional to the total value of the underlying transacted:

$$
\tau=\mathrm{S}(\mathrm{t}) \mathrm{n}(\mathrm{t}) \mathrm{k}
$$

where $n(t)$ is the number of units (e.g. shares for equities) bought or sold at time $t$ and $k / 2$ is the transaction cost for one share (sold or bought).

Under our replicating portfolio approach, to model $\delta \mathrm{C}$ with transaction costs we apply Leland's model:

$$
\delta \mathrm{C} \approx \Delta \delta \mathrm{S}(\mathrm{t})-\mathrm{rB}(\mathrm{t}) \delta \mathrm{t}-\frac{\mathrm{k}}{2}|\delta \Delta| \mathrm{S}(\mathrm{t})
$$

where the last term represents the transaction cost in our model. It has been shown by Leland that

$$
\frac{k}{2}|\delta \Delta| S(t) \approx \frac{\sigma^{2}}{2} \bar{L} S(t)^{2} \gamma \delta t
$$

where the Leland number $\bar{L}$ is

$$
\bar{L}=\sqrt{\left(\frac{2}{\pi}\right)}\left(\frac{k}{\sigma \sqrt{\delta t}}\right) .
$$

Hence our option risk model with liquidity risk is

$$
\rho(\delta \mathrm{C}) \approx \Delta \rho(\delta \mathrm{S}(\mathrm{t}))+\mathrm{rB}(\mathrm{t}) \delta \mathrm{t}+\frac{\sigma \mathrm{k}}{\sqrt{2 \pi}} \mathrm{S}(\mathrm{t})^{2} \gamma \sqrt{\delta \mathrm{t}}
$$

We note from equation (19) that in order to measure option risk with liquidity risk there is no significant increase in the level of computation. This is because the last term in equation (19) is not a function of $S(t+\delta t)$ but $S(t)$; hence it does not require recalculation for each simulated $S(t+\delta t)$. We also notice from equation (19) that the option risk measurement with liquidity risk does not require significant parameter estimation. In fact, most of the parameters contributing to the transaction costs can be observed or calculated from observable variables. Furthermore, Leland (1985) model and the replicating portfolio method are both derived without admitting arbitrage opportunities, which is important to model risk and preventing internal arbitrage opportunities (see for instance Erel et al. (2015)). 


\section{Numerical Experiments}

In this section we conduct numerical experiments to demonstrate and validate the replicating portfolio method of measuring option risk. To gauge the performance of the replicating portfolio method we also conducted numerical experiments on the Delta method to act as a fair benchmark. In this section first we explain our method, present the results of our experiments and then discuss them.

\subsection{Method}

In this section we conducted two numerical experiments. Firstly, we conducted a numerical experiment to measure the computation time of the replicating portfolio method against the Delta and full valuation methods. Secondly we evaluated the accuracy of the Delta and replicating portfolio methods in determining changes in option prices. All the numerical experiments were executed on a $1.61 \mathrm{GHz}$ computer, with $992 \mathrm{MB}$ RAM, running Matlab version 6.5.

For the computation time experiment we measured the time taken to compute the distribution of the change in call option price $\delta \mathrm{C}$ under a Black-Scholes model. The time measured was for a $\delta \mathrm{C}$ distribution consisting of one million samples. To obtain one million sample points we required one million random samples of $\delta S=S(\delta t)-S(0)$. The $\delta S$ random samples were obtained by generating the distribution of $\mathrm{S}(\delta \mathrm{t})$ under the BlackScholes model (geometric Brownian motion).

Using the samples of $\delta \mathrm{S}$ we calculated $\delta \mathrm{C}$ : for the full valuation method we applied the method outlined in the Appendix, for the Delta method we used equation (1) and for the replicating portfolio method we used equation (3). We note that the choice of BlackScholes parameters K,T, etc. do not affect any of the computation times. The BlackScholes option pricing equation along with other Black-Scholes parameters (e.g. option delta) did not require implementation as they are already available in the Matlab financial toolbox. The entire experiment was repeated ten times to obtain an average value of computation times. The results are presented in the next section.

In the second experiment we compared the accuracy of the Delta method against the replicating portfolio method. This was done by calculating $\delta \mathrm{C}$ over one day as this represents a realistic time period over which institutions may wish to evaluate the risk of options (although any time period could have been chosen). We compared the replicating portfolio methods' accuracies over a range of $\mathrm{K}$ and $\sigma$; we chose our range of $\mathrm{K}$ for $|\mathrm{K} / \mathrm{S}(0)-1| \leq 10 \%$ to test well beyond the range of actively traded options; the range of $\mathrm{K}$ for actively traded options tend to be within a range of $|\mathrm{K} / \mathrm{S}(0)-1| \leq 3 \%$ (see Fouque et al. (2000)) and beyond this range option prices tend to suffer from significant liquidity effects (see Fouque et al. (2000)). We also tested a range of volatility values $\sigma$ from $5 \%$ to $20 \%$. The typical volatility for an index is $\sigma=10 \%$, with $\sigma=20 \%$ considered to be high volatility (possibly occurring during a financial crisis), hence our testing ranges are robust well beyond standard ranges.

The range of $\mathrm{dS} / \mathrm{S}$ was chosen to be $\pm 1 \%, \pm 2 \%$ and $\pm 5 \%$ to reflect possible price changes in the underlying under different scenarios. Since a $10 \%$ return is the average return over one year for an index (see Hull (2000)), a range of $\mathrm{dS} / \mathrm{S}$ of $\pm 5 \%$ in one day reflects a scenario of a large price change. A $\pm 1 \%$ price change would be considered a normal price change and so reflects a typical price change scenario. $\mathrm{A} \pm 2 \%$ change would be considered a significant change, although a possible scenario. All scenarios were tested for robustness of our method.

We chose our option to have parameters $r=5 \%, T=100$ days, $S(0)=1000$ (although any values could have been chosen), as these are representative of typical option parameter 
values. We compared the accuracy of each method using the percentage relative error, taking the full valuation method as our correct answer. For example, for the Delta method the percentage relative error was calculated as

$$
\frac{\left|v_{F V M}-v_{D}\right|}{\left|v_{F V M}\right|} \times 100,
$$

where $\mathrm{v}_{\mathrm{FVM}}$ is the $\delta \mathrm{C}$ calculated by the full valuation method and $\mathrm{v}_{\mathrm{D}}$ was $\delta \mathrm{C}$ calculated by the Delta method. A similar equation was applied to the replicating portfolio method. To calculate the average relative percentage error we took the average of these results over 1000 samples for each $\mathrm{K}$ and $\sigma$.

\subsection{Results}

Table 1: Computation Time for Calculating $\delta C$ (In Seconds)

\begin{tabular}{|c|c|c|c|}
\hline Experiment & Delta Method & $\begin{array}{c}\text { Replicating Portfolio } \\
\text { Method }\end{array}$ & Full Valuation Method \\
\hline 1 & 166.375 & 166.812 & 504.312 \\
2 & 167.609 & 166.578 & 508.312 \\
3 & 165.485 & 168.000 & 510.297 \\
4 & 167.734 & 168.078 & 510.250 \\
5 & 167.453 & 167.453 & 507.469 \\
6 & 167.282 & 167.437 & 533.313 \\
7 & 167.469 & 167.906 & 512.562 \\
8 & 169.437 & 168.546 & 532.890 \\
9 & 169.656 & 166.859 & 510.015 \\
10 & 167.657 & 166.703 & 511.984 \\
\hline Average & 167.416 & 167.437 & 514.140 \\
\hline
\end{tabular}

Table 2: Average Relative Percentage Error for $\pm 1 \%$ Stock Range

\begin{tabular}{|c|c|c|c|c|c|c|c|c|}
\hline \multirow{2}{*}{$\begin{array}{c}\text { Strike } \\
\mathrm{K}\end{array}$} & \multicolumn{2}{|c|}{$\sigma=5 \%$} & \multicolumn{2}{c|}{$\sigma=10 \%$} & \multicolumn{2}{c|}{$\sigma=15 \%$} & \multicolumn{2}{c|}{$\sigma=20 \%$} \\
\cline { 2 - 9 } & $\mathrm{R}$ & $\mathrm{D}$ & $\mathrm{R}$ & $\mathrm{D}$ & $\mathrm{R}$ & $\mathrm{D}$ & $\mathrm{R}$ & $\mathrm{D}$ \\
\hline 900 & 0.00 & 15.60 & 1.25 & 15.46 & 6.20 & 20.43 & 16.29 & 33.18 \\
950 & 0.48 & 15.26 & 5.96 & 21.61 & 13.34 & 27.99 & 24.01 & 39.43 \\
1000 & 15.84 & 53.68 & 14.55 & 29.40 & 24.21 & 39.76 & 38.58 & 55.57 \\
1050 & 25.89 & 40.62 & 27.20 & 42.21 & 38.30 & 54.78 & 46.78 & 62.15 \\
1100 & 45.79 & 58.99 & 47.61 & 64.87 & 68.79 & 90.29 & 136.94 & 171.62 \\
\hline
\end{tabular}


Table 3: Average Relative Percentage Error for $\pm 2 \%$ Stock Range

\begin{tabular}{|c|c|c|c|c|c|c|c|c|}
\hline \multirow{2}{*}{$\begin{array}{c}\text { Strike } \\
\mathrm{K}\end{array}$} & \multicolumn{2}{|c|}{$\sigma=5 \%$} & \multicolumn{2}{c|}{$\sigma=10 \%$} & \multicolumn{2}{c|}{$\sigma=15 \%$} & \multicolumn{2}{c|}{$\sigma=20 \%$} \\
\cline { 2 - 9 } & $\mathrm{R}$ & $\mathrm{D}$ & $\mathrm{R}$ & $\mathrm{D}$ & $\mathrm{R}$ & $\mathrm{D}$ & $\mathrm{R}$ & $\mathrm{D}$ \\
\hline 900 & 0.00 & 7.27 & 0.91 & 8.26 & 3.32 & 9.97 & 6.35 & 12.84 \\
950 & 0.61 & 7.84 & 3.68 & 9.82 & 7.82 & 15.08 & 10.74 & 17.38 \\
1000 & 8.30 & 13.35 & 9.47 & 15.53 & 14.89 & 22.94 & 24.19 & 34.42 \\
1050 & 27.57 & 32.26 & 17.40 & 23.37 & 24.41 & 33.28 & 26.64 & 34.96 \\
1100 & 52.05 & 56.61 & 37.78 & 47.86 & 24.98 & 31.39 & 111.86 & 139.58 \\
\hline
\end{tabular}

Table 4: Average Relative Percentage Error for $\pm 5 \%$ Stock Range

\begin{tabular}{|c|c|c|c|c|c|c|c|c|}
\hline \multirow{2}{*}{ Strike } & \multicolumn{2}{|c|}{$\sigma=5 \%$} & \multicolumn{2}{c|}{$\sigma=10 \%$} & \multicolumn{2}{c|}{$\sigma=15 \%$} & \multicolumn{2}{c|}{$\sigma=20 \%$} \\
\cline { 2 - 9 } $\mathrm{K}$ & $\mathrm{R}$ & $\mathrm{D}$ & $\mathrm{R}$ & $\mathrm{D}$ & $\mathrm{R}$ & $\mathrm{D}$ & $\mathrm{R}$ & $\mathrm{D}$ \\
\hline 900 & 0.02 & 3.09 & 1.39 & 4.86 & 2.92 & 4.82 & 4.21 & 6.22 \\
950 & 1.86 & 5.10 & 5.26 & 7.00 & 6.28 & 8.14 & 6.96 & 8.94 \\
1000 & 19.74 & 21.05 & 13.46 & 15.32 & 12.31 & 14.98 & 20.56 & 29.90 \\
1050 & 63.38 & 64.71 & 24.51 & 26.19 & 16.58 & 18.44 & 14.16 & 16.17 \\
1100 & 117.07 & 118.13 & 38.89 & 41.10 & 24.55 & 26.95 & 19.79 & 22.20 \\
\hline
\end{tabular}

Note: R denotes the replicating portfolio method and D denotes the Delta method.

\subsection{Analysis}

The numerical experiments in Table 1 demonstrate that full valuation is computationally far more expensive than the Delta method; it takes approximately three times as long. The experiments also confirm that the replicating portfolio method is significantly less time consuming (computationally) than the full valuation method, in fact its computation time is practically identical to the Delta method.

It is worth noting in passing that in the past 20 years trading has become increasingly dominated by automated trading in many markets. Consequently, many trades are opened and closed on scales of the order of milliseconds (for example algorithmic trading). Hence even marginal improvements in computing times can make the difference between profit or loss trades. Therefore the significant computational time improvement by the replicating portfolio method is beneficial.

We expect both the Delta and replicating portfolio methods to have far lower computation times than the full valuation method because they require practically no calculation of nonlinear functions (other than for the one-off calculation of the option's Delta). The full valuation on the other hand must calculate the Black-Scholes equation (highly nonlinear function) for each sampled stock price. The replicating portfolio method therefore provides a significant saving in computation time, at a time comparable to the Delta method.

The savings in computation time become particularly important as we increase the number of options in a portfolio and the frequency with which the portfolio is valued during the day. Hence it can be seen that the full valuation method becomes increasingly impractical compared to the Delta and replicating portfolio methods. Additionally, we have used the Black-Scholes model to value the options, for which there exist many 
optimised computational implementations. For other option pricing models (e.g. with different underlying processes) the full valuation method will increase computation time.

The numerical experiments in Tables 2-4 demonstrate that the replicating portfolio is more accurate than the Delta method and most importantly, this is achieved with little additional computation time. The numerical experiments demonstrate that the replicating portfolio outperforms the Delta method over all $\mathrm{K}, \sigma$ and price ranges. This is because the additional terms obtained from the replicating portfolio argument (and are not present in the Delta method) enable more accurate modelling. To be more specific, the replicating portfolio takes into account the change in option price more accurately due to taking into account the impact of the option variables time and riskless rate. This is not achieved in the Delta method.

We observe that both the Delta and replicating portfolio increase in error as we increase with $\mathrm{K}$. This is because the Black-Scholes call Delta is $\Delta=\Psi\left(d_{1}\right)$, therefore $\Delta(\mathrm{t})$ decreases as $\mathrm{K}$ increases. As we multiply $\mathrm{dS}$ by $\Delta(\mathrm{t})$ in both the Delta method and replicating portfolio equation, a reduction in $\Delta(\mathrm{t})$ has the effect of changes in stock price being unable to model changes in option price. However, it should be noted in all cases the replicating portfolio method still provides a lower error than the Delta method.

The effect of increasing $\sigma$ generally has the impact of increasing the error of both the Delta and replicating portfolio method. However the influence of $\sigma$ is less predictable due to its relation with $\Delta(\mathrm{t})$. Since $\Delta=\Psi\left(d_{1}\right)$ for call options it can be shown that $\sigma$ has no monotonic increasing or decreasing relation with $\Delta(\mathrm{t})$ (whilst all other parameters are kept constant). Moreover, if we vary $\mathrm{K}$ and $\sigma$ then both will affect $\Delta(\mathrm{t})$, which in turn will affect the accuracies of our method.

The numerical experiments show that the replicating portfolio method is more accurate over all K, $\sigma$ and price ranges than the Delta method. In portfolios containing a range of options at different $\mathrm{K}$ (e.g. option trading strategies or static replicating portfolios) therefore the replicating portfolio offers a more accurate modelling method than the Delta method. Additionally, the replicating portfolio method does not admit arbitrage opportunities to occur in the modelling, unlike the Delta method. Most importantly, all these advantages are achieved without increasing computation cost, which is the main purpose behind such methods. Hence our replicating portfolio method is better suited to valuing portfolios of options than the Delta method.

In conclusion our numerical experiments show that our computational method is significantly faster than the full valuation method and has a computation time comparable to the delta method, demonstrating the fast computation time of our method. Secondly, our computation method is more accurate than the delta method for a range of volatilities, strikes and expiries. Hence the negligible increase in computation time using our computation method is worthwhile given the significant gain in accuracy. Additionally, our method does not admit arbitrage opportunities and other model risk errors.

\section{Conclusion}

In this paper we have proposed a new method of measuring option risk using the replicating portfolio method. We have shown that this method provides a fast computation of options by practically eliminating the requirement for evaluating nonlinear functions. This has resulted in computation times that are practically identical to the Delta method.

We have shown that the replicating portfolio approach provides many significant model risk advantages. Unlike prior models, one key advantage is that the replicating portfolio method does not allow arbitrage opportunities for complex portfolios of options. 
Furthermore, our model has lower calibration risk (only requiring observable market data to be implemented (except $\Delta(\mathrm{t}))$ ), it has parsimonious implementation and fewer model assumptions compared to competing models, which reduces implementation risk. Unlike competing methods, the replicating portfolio method can be applied to other models to take into account important model risk factors (e.g. liquidity risk and model misspecification). Another key advantage is that our method enables linear optimisation of portfolios containing options.

We conducted numerical experiments on our replicating portfolio method to validate our method. These results have demonstrated that the replicating portfolio method computes changes in option prices in times practically identical to those of the Delta method whilst also giving lower relative error. In conclusion, we believe the replicating portfolio offers significant modelling and computational advantages over alternative modelling methods and this will be of interest to industry professionals.

In terms of future possible areas of research, this should involve developing the computational method for other contingent claims, such as exotic options, energy options and barrier options. Another area for future research is to develop the replicating portfolio method with relaxed Black-Scholes modelling assumptions, such as the explicit inclusion of taxes, dividends and stochastic interest rates. Finally, the risk measurement method could be extended to real options analyses, as risk measurement is important for corporate finance applications. 


\section{Appendix}

\section{1: Black-Scholes Equation}

The Black-Scholes option pricing model is given by

$$
\begin{gathered}
C(S(t), t, T, r, \sigma, K)=S(t) \Psi\left(d_{1}\right)-K e^{-r(T-t)} \Psi\left(d_{2}\right) \\
\text { where } d_{1}=\frac{\ln (S(t) / K)+\left(r+\frac{1}{2} \sigma^{2}\right)(T-t)}{\sigma \sqrt{(T-t)}}, \\
d_{2}=\frac{\ln (S(t) / K)+\left(r-\frac{1}{2} \sigma^{2}\right)(T-t)}{\sigma \sqrt{(T-t)}}, \\
=d_{1}-\sigma \sqrt{(T-t)} .
\end{gathered}
$$

In $\mathrm{C}(\mathrm{S}(\mathrm{t}), \mathrm{t}, \mathrm{T}, \mathrm{r}, \sigma, \mathrm{K}) \mathrm{t}$ is the time at which $\mathrm{C}$ is being priced, $\mathrm{T}$ is the expiration date, $\Psi(\cdot)$ is the standard normal cumulative distribution function and $\mathrm{K}$ is the strike price.

\section{2: Algorithm for Full Valuation Method}

Let index $i=\{1,2, . ., n\}$ where $\mathrm{n}$ is the number of Monte Carlo simulations.

1. Calculate initial option price $\mathrm{C}(0)$ : $\mathrm{C}(\mathrm{S}(0), \mathrm{t}, \mathrm{T}, \mathrm{r}, \sigma, \mathrm{K})$.

Set $\mathrm{i}=1$.

2. Simulate $S(\delta t)$ from $\mathrm{S}(0)$.

Denote simulated value for iteration i as $S_{i}(\delta t)$.

3. Calculate option price $C_{i}(\delta t)$ using $S_{i}(\delta t): \mathrm{C}_{\mathrm{i}}\left(\mathrm{S}_{\mathrm{i}}(\delta t), \delta t, \mathrm{~T}, \mathrm{r}, \sigma, \mathrm{K}\right)$.

4. Calculate loss: $\mathrm{C}(0)-C_{i}(\delta t)$.

5. Increment $i$.

If $\mathrm{i}=\mathrm{n}+1$ then stop, otherwise goto step 2 .

\section{3: Bond Price Equation}

The price of a bond $\mathrm{B}(\mathrm{t})$ is given by Bjork (2004)

$$
\begin{aligned}
& B(t)=B(0) \exp \left(\begin{array}{l}
t \\
\int \\
0
\end{array} d t\right), \\
& d B=r B(t) d t .
\end{aligned}
$$

\section{4: Arbitrage Definition}

An arbitrage possibility in a financial market is a portfolio $V(t)$ such that:

- $V(0) \leq 0$;

- $V(T) \geq 0$ almost surely and

- $E[V(T)] \geq 0$. 


\section{5: Expressions for Call Option Terms}

For a call option it can be shown that (see Baxter and Rennie (1996)):

$$
\begin{aligned}
\phi_{1}(t) & =\Delta(t), \\
B(t) \phi_{2}(t) & =K e^{-r(T-t)} \Psi\left(d_{2}\right) .
\end{aligned}
$$

Alternatively we can express $B(t) \phi_{2}(t)$ as

$$
\begin{aligned}
B(t) \phi_{2}(t) & =\Delta S(t)-C(t), \\
& =\phi_{1}(t) S(t)-C(t) .
\end{aligned}
$$

\section{6: Proof for Portfolio with Options}

$$
\delta D(t) \approx(n+\Delta) \delta S(t)+\phi_{2} \delta B
$$

We have

$$
\begin{aligned}
\delta D(t) & \approx n \delta S(t)+\delta C(S(t)), \\
& \approx n \delta S(t)+\phi_{1} d S+\phi_{2} \delta B, \\
& \approx\left(n+\phi_{1}\right) \delta S(t)+\phi_{2} \delta B, \\
& \approx(n+\Delta) \delta S(t)+\phi_{2} \delta B .
\end{aligned}
$$

\section{7: Proof of Put-Call Parity of Replicating Portfolio Method}

A put option can be replicated by $\Delta_{p}$ units of shares and a long position in $\phi_{2 p}(t)$ units in bonds.

$$
\begin{gathered}
P=\Delta_{p} S(t)+\phi_{2 p}(t) B(t), \\
d P=\Delta_{p} d S(t)+\phi_{2 p}(t) d B(t), \\
\delta P \approx \Delta_{p} \delta S(t)+\phi_{2 p}(t) r B(t) \delta t(t) .
\end{gathered}
$$

We also have

$$
\begin{gathered}
\phi_{2 p}(t) B(t)=K e^{-r(T-t)}-\phi_{2}(t) B(t), \\
\phi_{2 p}(t) d B(t)=d\left(K e^{-r(T-t)}\right)-\phi_{2}(t) d B(t) .
\end{gathered}
$$

By put-call parity we have

$$
\begin{aligned}
\delta P & \approx \delta C+\delta\left(K e^{-r(T-t)}\right)-\delta S, \\
& \approx\left(\Delta \delta S-\phi_{2}(t) \delta B(t)\right)+\delta\left(K e^{-r(T-t)}\right)-\delta S, \\
& \approx \Delta_{p} \delta S+\left(\delta\left(K e^{-r(T-t)}\right)-\phi_{2}(t) \delta B(t)\right),
\end{aligned}
$$




$$
\approx \Delta_{p} \delta S+\phi_{2 p}(t) \delta B(t)
$$

Hence equations (28) and (27) are equal. Therefore the put-call parity is obeyed.

\section{8: Coherency Axioms for Risk Measurement}

A risk measure $\rho($.$) is coherent if it is:$

1. monotonic: if $Z_{1} \leq Z_{2}$ then $\rho\left(Z_{1}\right) \geq \rho\left(Z_{2}\right)$;

2. homogeneous: $\rho\left(\kappa Z_{1}\right)=\kappa \rho\left(Z_{1}\right)$, where $\kappa \in \mathbf{R}^{+}$is a positive constant;

3. translation invariant: $\rho\left(Z_{1}+v\right)=\rho\left(Z_{1}\right)-v$, where $v \in \mathbf{R}$ is a constant (or a riskless bond portfolio);

4. subadditive: $\rho\left(Z_{1}+Z_{2}\right) \leq \rho\left(Z_{1}\right)+\rho\left(Z_{2}\right)$.

\section{9: Put-Call Parity}

The put-call parity theorem states that, in an arbitrage free market, a call $\mathrm{C}(\mathrm{S}(\mathrm{t}), \mathrm{t}, \mathrm{T}, \mathrm{r}, \mathrm{K})$ and put $\mathrm{P}(\mathrm{S}(\mathrm{t}), \mathrm{t}, \mathrm{T}, \mathrm{r}, \mathrm{K})$ with the same $\mathrm{S}(\mathrm{t}), \mathrm{K}$ and $\mathrm{T}$ obey the relation:

$$
\mathrm{P}(\mathrm{S}(\mathrm{t}), \mathrm{t}, \mathrm{Tr}, \mathrm{K})=\mathrm{Ke} \mathrm{e}^{-\mathrm{r}(\mathrm{T}-\mathrm{t})}-\mathrm{S}(\mathrm{t})+\mathrm{C}(\mathrm{S}(\mathrm{t}), \mathrm{t}, \mathrm{T}, \mathrm{r}, \mathrm{K})
$$

\subsection{0: Example of Internal Arbitrage By Put-Call Parity}

Let us assume there are 2 departments in 1 company, $\mathrm{H} 1$ and H2. Department H1 creates a portfolio $\mathrm{M}$ consisting of buying put option $\mathrm{P}$, and short selling a call option $\mathrm{C}$. Hence:

and

$$
M=P-C,
$$

$$
\delta M=\delta P-\delta C
$$

Both $\mathrm{P}$ and $\mathrm{C}$ are identical in terms of option parameters, that is $\mathrm{T}, \mathrm{r}, \mathrm{K}, \sigma, \mathrm{S}(\mathrm{t})$ etc.. By the Delta-Gamma method:

$$
\delta P \approx \Delta_{p} \delta S+\frac{\gamma^{2}}{2}(\delta S)^{2}
$$

where $\Delta_{p}$ is the option delta for a put and $\delta C$ is

$$
\begin{aligned}
\delta C & \approx \Delta \delta S+\frac{\gamma^{2}}{2}(\delta S)^{2}, \\
& \approx\left(\Delta_{p}+1\right) \delta S+\frac{\gamma^{2}}{2}(\delta S)^{2} .
\end{aligned}
$$

The previous line is possible because in any option pricing model it is known that $\Delta_{=} \Delta_{p}+1$ . Therefore

$$
\delta M=\delta P-\delta C
$$




$$
=\left(\Delta_{p} \delta S+\frac{\gamma^{2}}{2}(\delta S)^{2}\right)-\left(\left(\Delta_{p}+1\right) \delta S+\frac{\gamma^{2}}{2}(\delta S)^{2}\right),
$$

$=-\delta S$.

Hence department $\mathrm{H} 1$ expects $-\delta S$ payoff from its portfolio M. Now department $\mathrm{H} 2$ can sell the portfolio M to department H1. By the put-call parity:

$$
\begin{aligned}
P & =K e^{-r(T-t)}-S(t)+C, \\
P-C & =K e^{-r(T-t)}-S(t) .
\end{aligned}
$$

So H2's payout from the above portfolio will be:

$$
\begin{aligned}
& d P-d C=d\left(K e^{-r(T-t)}\right)-d S \\
& \delta P-\delta C \approx K e^{-r(T-\delta t)}-\delta S
\end{aligned}
$$

Now $\mathrm{H} 2$ will only need to pay out $-\delta S$ to $\mathrm{H} 1$ for the portfolio it sold to $\mathrm{H} 1$ because $\mathrm{H} 1$ is expecting $-\delta S$ from its model. However the correct value of the portfolio is given in the last equation. Hence using the last equation we see that $\mathrm{H} 2$ will always be able to make a riskless profit of $K e^{-r(T-\delta t)}$, regardless of the value of $\mathrm{S}(\mathrm{t})$. Hence this represents an arbitrage opportunity. 


\section{References}

Acharya, V. V., Schaefer, S., \& Zhang, Y. (2015). Liquidity risk and correlation risk: A clinical study of the General Motors and Ford Downgrade of May 2005. Quarterly Journal of Finance, 5(02).

Alexander, C. (2001), Market models, John Wiley \& Sons.

Alexander, C. and Sarabia, J. M. (2012), "Quantile uncertainty and value-at-risk model risk”, Risk Analysis , Vol. 32, Wiley Online Library, pp. 1293-1308.

Artzner, P., Delbaen, F., Eber, J. and Heath, D. (1997), "Thinking coherently", Risk, Vol. 10, pp. 68-71.

Avellaneda, M., Levy, A. and Paras, A. (1995), "Pricing and hedging derivative securities in markets with uncertain volatilities", Applied Mathematical Finance, Vol. 2, Routledge, pp. 73-88.

Baxter, M. and Rennie, A. (1996), Financial Calculus: an introduction to derivative pricing, Cambridge University Press.

Björk, T. (2004), Arbitrage Theory in Continuous Time, Oxford University Press.

Black, F. and Scholes, M. (1973), "The Pricing of Options and Corporate Liabilities", The Journal of Political Economy , Vol. 81, JSTOR, pp. 637-654.

Britten-Jones, M. and Schaefer, S. (1998), "Non-linear value-at-risk", European Finance Review, Vol. 2, Springer, pp. 161-187.

Broda, S. A. (2012), "The expected shortfall of quadratic portfolios with heavytailed risk factors", Mathematical Finance, Vol. 22, Wiley Online Library, pp. 710-728.

Christoffersen, P. (2003), Elements of financial risk management, Academic Pr.

Derman, E., Ergener, D. and Kani, I. (1995), "Static options replication", The Journal of Derivatives , Vol. 2, Institutional Investor Journals, pp. 78-95.

Dowd, K. (2011), An Introduction to Market Risk Measurement, Wiley Finance.

El-Jahel, L., Perraudin, W. and Sellin, P. (1999), "Value at risk for derivatives", The Journal of Derivatives , Vol. 6, Institutional Investor Journals, pp. 7-26.

Erel, I., Myers, S. C., \& Read, J. A. (2015). A theory of risk capital. Journal of Financial Economics, 118(3), 620-635.

Fouque, J., Papanicolaou, G. and Sircar, K. (2000), Derivatives in Financial Markets with Stochastic Volatility, Cambridge University Press New York. 
Glasserman, P., Heidelberger, P. and Shahabuddin, P. (2001), "Efficient Monte Carlo methods for value-at-risk", Mastering Risk, Vol. 2, pp. 5-17.

Goovaerts, M. J., Kaas, R., Dhaene, J., \& Tang, Q. (2004). Some new classes of consistent risk measures. Insurance: Mathematics and Economics,34(3), 505-516.

Guillaume, F. and Schoutens, W. (2012), "Calibration risk: Illustrating the impact of calibration risk under the heston model", Review of Derivatives Research , Vol. 15, Springer, pp. 57-79.

Hao, F. and Yang, H. (2011), "Coherent risk measure for derivatives under black- scholes economy with regime switching", Managerial Finance, Vol. 37, Emerald Group Publishing Limited, pp. 1011-1024.

Hull, J. (2000), Options, futures and other derivatives, Prentice Hall New Jersey. Jarrow, R. and Turnbull, S. (1996), Derivative securities, South-Western College Pub.

Jaschke, S. (2002), "The Cornish-Fisher expansion in the context of deltagamma- normal approximations", Journal of Risk, Vol. 4.

Kabir, M. and Hassan, M. (2005), "The Near-Collapse of LTCM, US Financial Stock Returns, and the Fed", Journal of Banking and Finance , Vol. 29, Elsevier, pp. 441-460.

Kerkhof, J., Melenberg, B. and Schumacher, H. (2010), "Model risk and capital re-serves", Journal of Banking \& Finance, Vol. 34, Elsevier, pp. 267-279.

Kondo, H. and Saito, S. (2012), "Bayesian approach to measuring parameter and model risk in loss ratio estimation", Journal of Math-for-Industry , Vol. 4, pp. 8589.

Krakovsky, A. (1999), "Pricing liquidity into derivatives", Risk , Vol. 12, p. 65067.

Kwok, Y. (1998), Mathematical models of financial derivatives, Springer New York.

Leland, H. (1985), "Option pricing and replication with transactions costs", Journal of Finance , American Finance Association, pp. 1283-1301.

Ma, J., Deng, D., \& Zheng, H. (2016). Convergence analysis and optimal strike choice for static hedges of general path-independent pay-offs.Quantitative Finance, 16(4), 593 603.

Schmeiser, H., Siegel, C. and Wagner, J. (2012), "The risk of model misspecification and its impact on solvency measurement in the insurance sector", Journal of Risk Finance, The , Vol. 13, Emerald Group Publishing Limited, pp. 285-308.

Siven, J., Lins, J. and Szymkowiak-Have, A. (2009), "Value-at-Risk 
computation by Fourier inversion with explicit error bounds", Finance Research Letters , Vol. 6, Elsevier, pp. 95-105.

Sorwar, G. and Dowd, K. (2010), "Estimating financial risk measures for options", Journal of Banking \& Finance , Vol. 34, Elsevier, pp. 1982-1992.

Szegö, G. (2005), “Measures of risk”, European Journal of Operational Research , Vol.163, Elsevier, pp. 5-19.

Wilmott, P. et al. (1998), Derivatives: the theory and practice of financial engineering, John Wiley \& Sons. 